\title{
Prevalence and Epidemiological Factors Involved in Cellulitis in Korean Patients With Lymphedema
}

\author{
Sae In Park, $\mathrm{MD}^{1}$, Eun Joo Yang, $\mathrm{MD}^{2}$, Dong Kyu Kim, $\mathrm{MD}^{1}$, Ho Joong Jeong, MD¹, \\ Ghi Chan Kim, MD', Young-Joo Sim, $\mathrm{MD}^{1}$
}

\begin{abstract}
${ }^{1}$ Department of Physical Medicine and Rehabilitation, Kosin University College of Medicine, Busan; ${ }^{2}$ Department of Physical Medicine and Rehabilitation, Seoul National University Bundang Hospital, Seongnam, Korea
\end{abstract}

Objective To evaluate the prevalence and associated factors involved in cellulitis with lymphangitis among a group of Korean patients who were being treated for lymphedema. We present our epidemiologic research and we also report a systematic review of these types of cases.

Methods This was a retrospective medical record study among 1,246 patients diagnosed with lymphedema. The study was carried out between January 2006 and December 2012 at the Kosin University Gospel Hospital and Seoul National University Bundang Hospital. Cases were examined for onset time, affected site, seasonal trend, and recurrence pattern of lymphedema, lymphangitis, and cellulitis. We also evaluated the history of blood-cell culture and antibiotic use.

Results Ninety-nine lymphedema patients experienced complications such as cellulitis with accompanying lymphangitis. Forty-nine patients had more than two recurrences of cellulitis with lymphangitis. The incidence and recurrence of cellulitis with lymphangitis were significantly higher in the patients with lower-extremity lymphedema. There was a significant trend toward higher cellulitis prevalence in the lower-extremity lymphedema group according to the time of lymphedema onset. Among the cellulitis with lymphangitis cases, 62 cases were diagnosed through blood-cell culture; 8 of these 62 cultures were positive for $\beta$-hemolytic streptococci.

Conclusion The prevalence rate of cellulitis with lymphangitis in patients with lymphedema was $7.95 \%$, and the prevalence of recurrent episodes was $3.93 \%$. Especially, there was high risk of cellulitis with lymphangitis after occurrence of lower-extremity lymphedema with passage of time. Lymphedema patients should be fully briefed about the associated risks of cellulitis before treatment, and physicians should be prepared to provide appropriate preventive education.

Keywords Cellulitis, Lymphangitis, Lymphedema

Received May 20, 2015; Accepted September 8, 2015

Corresponding author: Young-Joo Sim

Department of Physical Medicine and Rehabilitation, Kosin University College of Medicine, 262 Gamcheon-ro, Seo-gu, Busan 49267, Korea

Tel: +82-51-990-6156, Fax: +82-51-241-2019, E-mail: oggum@hanmail.net

(c) This is an open-access article distributed under the terms of the Creative Commons Attribution Non-Commercial License (http://creativecommons.org/ licenses/by-nc/4.0) which permits unrestricted noncommercial use, distribution, and reproduction in any medium, provided the original work is properly cited. Copyright () 2016 by Korean Academy of Rehabilitation Medicine 


\section{INTRODUCTION}

Lymphedema can be classified into primary lymphedema, which is caused by formation of abnormal lymphatic vessels, and secondary lymphedema, which is caused by damage to the lymph vessels or lymph nodes. Primary lymphedema is a rare disease that is mainly observed in children, and it has a reported prevalence rate of 1.2 cases per 100,000 persons $<20$ years of age [1]. Secondary lymphedema accounts for more than $90 \%$ of global lymphedema cases $[2,3]$. There are several known causes in adults, such as infection and malignant tumors. Lymphedema is mainly associated with malignant tumors and often occurs after radiation therapy, operation, or direct/indirect pressure on the lymph vessels by cancerous tissue. Breast cancer-related lymphedema (BCRL), a complication that occurs during treatment, is reported to have a regional prevalence rate of $32.5 \%$ [4]. Since lymphedema can lead to secondary sequelae that impact the quality of life, particularly among patients who are being treated for gynecologic cancer and breast cancer, much attention has recently been paid to this health issue, and it is becoming an active area of research.

Cellulitis with lymphangitis occurs when abnormal proliferation of bacteria or toxin absorbed into wounds or skin infections spreads into subcutaneous tissue, where lymph fluid accumulates and leads to an invasive infection of muscle or mucosal tissue. Symptoms vary in severity, and cellulitis can cause different systemic complications, such as tachycardia, hypotension, or general malaise [5]. Typically, cellulitis is preceded by flu-like symptoms, and it is characterized by erythema and swelling that presents as a hot sensation or tenderness at the affected site [6]. These skin infections are mainly caused by $\beta$-hemolytic streptococci invasion into the extremities; $75 \%-90 \%$ of cellulitis cases occur in the lower limbs [7-12].

Lymphedema has been found to be an important risk factor for cellulitis with lymphangitis [13]. Although the epidemiology of lymphedema is well described, very few studies have explored the epidemiology of cellulitis with lymphangitis. A recent study reported that BCRL patients are twice as likely to have cellulitis compared to breast cancer patients without BCRL [14]. Another study found a $40 \%-50 \%$ prevalence rate of cellulitis among Asian patients with lymphedema [15]. Additional common risk factors for cellulitis include being overweight, damage to the cutaneous barrier, wound, venous insufficiency, and swelling of the lower extremities $[13,16]$. Treatment of these symptoms is empirically driven because epidemiologic understanding of multidirectional causes of cellulitis is lacking. As a consequence of this approach, patients have a high healthcare burden and cost due to treatment. Herein, we present our epidemiologic research on the prevalence rate of cellulitis with lymphangitis in lymphedema patients and we also report a systematic review of these types of cases in Korea, based on a patient sample from Kosin University Gospel Hospital and Seoul National University Bundang Hospital.

\section{MATERIALS AND METHODS}

\section{Study design}

Kosin University Gospel Hospital in Busan and Seoul National University Bundang Hospital in Seongnam conducted a retrospective medical record study of the epidemiology and prevalence of cellulitis including lymphangitis among patients diagnosed with lymphedema through outpatient and inpatient care from January 2006 to December 2012. Lymphedema diagnoses were based on the International Statistical Classification of Diseases and Related Health Problems, 10th revision (ICD-10) for patients with codes I890 and I972. Additionally, the medical record study of lymphangitis and cellulitis included ICD-10 codes I891 and L039 and cases with a history of antibiotic therapy along with swelling, tightness, erythema, and rubefaction. Although we categorized patients into different cellulitis-type groups on the basis of the ICD-10 code, some patients were excluded from the study due to insufficient information for analysis.

A total of 1,246 lymphedema patients were included, and 99 of these patients were confirmed to have developed cellulitis with lymphangitis. The patients were examined for onset time, affected site, annual trends, and recurrence patterns of lymphedema, cellulitis, and lymphangitis. We also investigated presumptive causes, specific associated cellulitis with lymphangitis symptoms, laboratory findings including blood-cell culture, and history of antibiotic therapy.

The study protocol was approved by the Ethical Review Committee for research involving human research subjects, Kosin University College of Medicine. 


\section{Data collection}

Patient data were extracted and recorded in a case report form (CRF). Parameters recorded in the CRF were 1) patient demographics including age, sex, body mass index (BMI); 2) lymphedema onset time, affected extremity (upper or lower), duration; 3) cellulitis with lymphangitis onset time, presumed cause, symptoms, recurrence pattern; 4) laboratory findings including blood-cell culture; and 5) classes and timeframes for oral or intravenous administration of antibiotics.

Patient age was recorded at the time of diagnosis of lymphedema and then again at the diagnosis of cellulitis with lymphangitis. BMI values were classified as either $\leq 25$ or $>25$ in order to distinguish between obese and non-obese patients. Onset time of lymphedema was recorded as the date of first diagnosis, regardless of whether it occurred in the outpatient or inpatient care. The presumed causes of cellulitis with lymphangitis were broadly divided into the following four categories: 1) iatrogenic cause (radiation therapy, chemotherapy), 2) infection (blood sampling, wound, common cold), 3) extreme exercise or labor (mountain climbing, jogging, laundry, mopping), 4) others and unclear causes. Common symptoms were divided into the following four broad categories: 1 ) acutely aggravated edema, 2) hot sensation or rubefaction, 3) pain, 4) others.

Laboratory samples were collected during the first observation of lymphangitis or cellulitis. Laboratory analyses included determination of white blood cell (WBC) count, erythrocyte sedimentation rate (ESR), and C-reactive protein (CRP) level in order to assess the inflammatory changes associated with cellulitis and lymphangitis. The normal ranges for these measurements were: WBC, 4,300-9,400/ $\mu \mathrm{L}$; ESR, 1-20 mm/hr; and CRP, 0-1 mg/dL.

\section{Statistical analysis}

All statistical analyses were conducted using SPSS ver. 14.0 for Windows (SPSS Inc., Chicago, IL, USA). The chisquare $\left(\chi^{2}\right)$ test was used to compare prevalence and recurrence rates of cellulitis with lymphangitis among all lymphedema patients, stratified by the affected extremity (upper or lower). Additionally, to compare inflammation and BMI among cellulitis with lymphangitis patients, stratified by the affected extremity, we used t-test and $\chi^{2}$ test to explore the differences in BMI values and laboratory results. A p-value $<0.05$ was considered statistically significant. Linear-by-linear association was used to estimate the likelihood of cellulitis with lymphangitis among lymphedema patients according to the onset duration. Onset values were rounded to the nearest month, and onset ratios were calculated to identify annual trends.

\section{RESULTS}

Among the 1,246 lymphedema patients enrolled in this study, 99 patients $(7.95 \%)$ were confirmed to have cellulitis complications, including lymphangitis. Stratified by age, $6.03 \%$ ( 7 of 116 ) of male patients and $8.14 \%$ $(92$ of 1,130$)$ of female patients developed cellulitis with lymphangitis. A total of 188 episodes of cellulitis with lymphangitis were observed in these 99 patients between 2006 and 2012.

Table 1 summarizes patient characteristics stratified by age, sex, and affected extremity. The mean age of the study population was 51 years (range, 15-81 years), and the mean BMI was $24.27 \mathrm{~kg} / \mathrm{m}^{2}$ (range, $16.77-40.58 \mathrm{~kg}$ / $\mathrm{m}^{2}$ ). When cellulitis with lymphangitis patients were stratified by affected extremity, there was no statistically significant difference with respect to mean BMI (upperextremity group, $23.11 \mathrm{~kg} / \mathrm{m}^{2}$; lower-extremity group, $24.99 \mathrm{~kg} / \mathrm{m}^{2}$ ). Moreover, on comparing cellulitis with lymphangitis episodes among patients with BMI $>25 \mathrm{~kg} / \mathrm{m}^{2}$, no significant difference was observed (upper-extremity group, 25 of the 76 episodes; lower-extremity group, 57 of the 112 episodes) (Table 2 ).

Table 1. Number of age- and sex-specific cases and total cellulitis $^{\text {a) }}$ with lymphangitis ${ }^{\text {b) }}$ cases among patients with lymphedema ${ }^{\text {c) }}$

\begin{tabular}{|c|c|c|c|c|c|}
\hline \multirow{2}{*}{$\begin{array}{c}\text { Age group } \\
\text { (yr) }\end{array}$} & \multicolumn{2}{|c|}{ Upper extremity } & \multicolumn{2}{|c|}{ Lower extremity } & \multirow{2}{*}{ Total } \\
\hline & $\mathbf{M}$ & $\mathbf{F}$ & M & $\mathbf{F}$ & \\
\hline$<40$ & 0 & 3 & 2 & 2 & 7 \\
\hline $40-49$ & 0 & 18 & 1 & 13 & 32 \\
\hline $50-59$ & 0 & 10 & 4 & 10 & 24 \\
\hline $60-69$ & 0 & 6 & 0 & 15 & 21 \\
\hline $70-79$ & 0 & 3 & 0 & 7 & 10 \\
\hline$\geq 80$ & 0 & 2 & 0 & 3 & 5 \\
\hline Total & 0 & 42 & 7 & 50 & 99 \\
\hline
\end{tabular}

$\mathrm{M}$, male; F, female.

${ }^{\text {a) }}$ Cellulitis (ICD-10 code: L039), b) lymphangitis (ICD-10

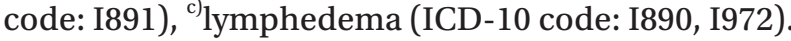


Table 2. Clinical presentation and characteristics of cellulitis ${ }^{\text {a) }}$ with lymphangitis ${ }^{\text {b) }}$ episodes in patients with lymphedema $^{\text {c) }}$

\begin{tabular}{ccccc}
\hline & $\begin{array}{c}\text { Upper extremity } \\
(\mathbf{n}=\mathbf{7 6})\end{array}$ & $\begin{array}{c}\text { Lower extremity } \\
(\mathbf{n}=\mathbf{1 1 2})\end{array}$ & $\begin{array}{c}\text { Total } \\
(\mathbf{n}=\mathbf{1 8 8})\end{array}$ & p-value \\
\hline BMI $\left(\mathrm{kg} / \mathrm{m}^{2}\right)$ & 23.11 & 24.99 & 24.27 & 0.102 \\
$>25$ & $25(32.9)$ & $57(50.9)$ & $82(43.6)$ & 0.123 \\
\hline Laboratory findings & & & & \\
WBC $(/ \mu \mathrm{L})$ & 8.54 & 9.43 & 9.02 & 0.275 \\
\hline$>9,400$ & $19(25.0)$ & $49(43.8)$ & $68(36.2)$ & $0.030^{*}$ \\
ESR $(\mathrm{mm} / \mathrm{hr})$ & 26.58 & 38.03 & 33.02 & 0.100 \\
\hline$>20$ & $27(35.5)$ & $64(57.1)$ & $91(48.4)$ & 0.065 \\
\hline CRP $(\mathrm{mg} / \mathrm{dL})$ & 2.35 & 7.33 & 5.38 & $0.006^{*}$ \\
\hline$>1$ & $24(31.6)$ & $62(55.4)$ & $86(45.7)$ & 0.070 \\
\hline
\end{tabular}

Values are presented as median or number (\%).

BMI, body mass index; WBC, white blood cell; ESR, erythrocyte sedimentation rate; CRP, C-reactive protein.

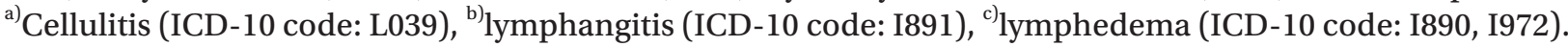
${ }^{*} \mathrm{p}<0.05$ (t-test or chi-square analysis).

Table 3. Prevalence and recurrence rates of cellulitis ${ }^{\text {a) }}$ with lymphangitis ${ }^{\text {b) }}$ in patients with lymphedema ${ }^{\text {c) }}$

\begin{tabular}{lcccc}
\hline & $\begin{array}{c}\text { Upper } \\
\text { extremity } \\
(\mathbf{n = 8 0 5})\end{array}$ & $\begin{array}{c}\text { Lower } \\
\text { extremity } \\
(\mathbf{n = 4 2 7})\end{array}$ & $\begin{array}{c}\text { Total } \\
(\mathbf{n = 1 , 2 4 6 )}\end{array}$ & p-value \\
\hline Cellulitis with lymphangitis cases & $42(5.2)$ & $57(13.3)$ & $99(8.0)$ & $<0.001^{*}$ \\
$\begin{array}{l}\text { Recurrent cases } \\
\text { Cellulitis cases stratified by time of lymphedema onset }\end{array}$ & $19(2.4)$ & $30(7.0)$ & $49(4.0)$ & $<0.001^{*}$ \\
\hline$<6$ months & $25(3.1)$ & $22(5.2)$ & $47(3.8)$ \\
\hline 6 months to 1 year & $3(0.4)$ & $2(0.5)$ & $5(0.4)$ \\
\hline to 1.5 years & $5(0.6)$ & $3(0.7)$ & $8(0.6)$ \\
\hline 1.5 to 2 years & $2(0.3)$ & $6(1.4)$ & $8(0.6)$ \\
\hline to 5 years & $6(0.8)$ & $9(2.1)$ & $15(1.2)$ \\
\hline 5 years & $1(0.1)$ & $15(3.5)$ & $16(1.3)$ \\
\hline
\end{tabular}

Values are presented as number (\%).

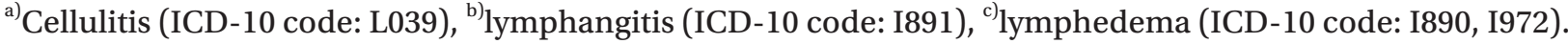

${ }^{*} \mathrm{p}<0.05$ (chi-square analysis).

On comparing cellulitis with lymphangitis risk according to the affected site, the incidence was significantly higher among patients with lymphedema in the lower extremities (Table 3). Meanwhile, 49 of the 99 cellulitis with lymphangitis patients experienced more than two recurrences, and these recurrences were significantly more common among patients with lower-extremity lymphedema.

On analyzing the 6-month period following lymphedema onset, more than half of the upper-extremity lymphedema cases showed a tendency for developing cellulitis with lymphangitis within 6 months since lymphedema occurrence $(59.52 \%, 25$ of the 42 cases) compared to the lower-extremity lymphedema cases that showed a tendency for developing cellulitis with lymphangitis after 6 months of lymphedema occurrence $(61.40 \%$, 35 of the 57 cases). Fig. 1 shows the number of cellulitis with lymphangitis occurrences within the two extremity groups according to the time elapsed since lymphedema occurrence. A significant trend toward a higher incidence of cellulitis with lymphangitis was demonstrated in patients with lower-extremity lymphedema with passage of time 


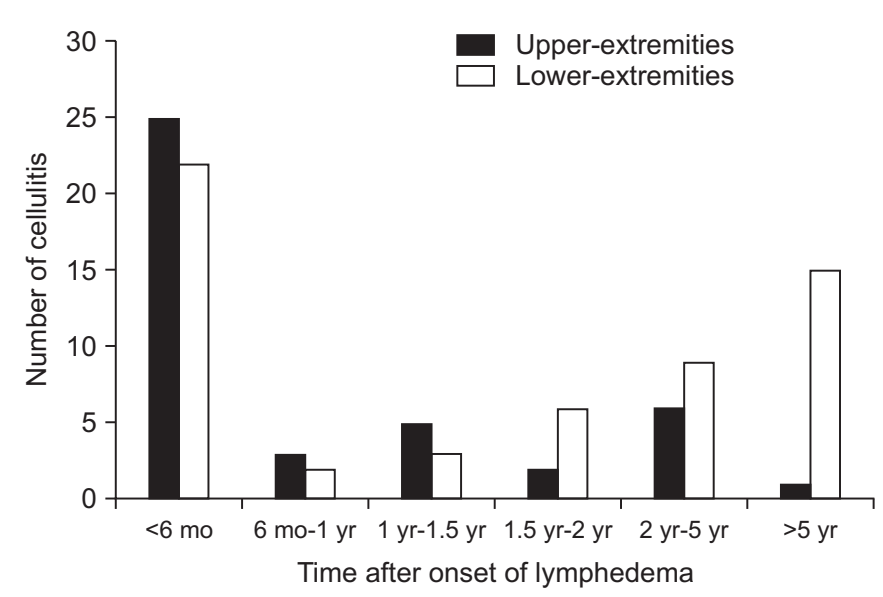

Fig. 1. Cellulitis ${ }^{\text {a) }}$ in the upper-extremity and lower-extremity groups according to onset time of lymphedema ${ }^{\text {b) }}$. A trend toward higher prevalence of cellulitis was demonstrated in the lower extremity group $(\mathrm{p}=0.002$, linear-

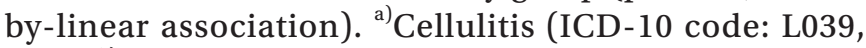

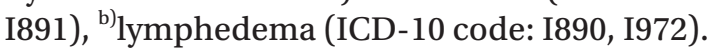

through a linear-by-linear association $(\mathrm{p}=0.002)$. Cellulitis with lymphangitis was most common during summer, when the relative risk was twice that in spring when cellulitis with lymphangitis was least common (Fig. 2).

In most cases, cellulitis including lymphangitis was of idiopathic or unknown origin (144 episodes among the 188 total episodes, $76.60 \%$ ); the next most common causes were infectious processes, such as common cold or wounds (24 episodes, 12.77\%), extreme exercise or labor (15 episodes, $7.98 \%$ ), and radiation therapy or chemotherapy (5 episodes, $2.66 \%$ ).

The major symptoms of cellulitis with lymphangitis were hot sensation and rubefaction (162 episodes among the 188 episodes, $86.17 \%$ ), followed by other symptoms of acutely aggravated edema (50 episodes, $26.60 \%$ ) and pain (23 episodes, $12.23 \%$ ). The above symptoms mostly presented in combination with other symptoms rather than in isolation.

Blood culture was performed in 62 cases of cellulitis with lymphangitis; 8 of these cases were positive for $\beta$-hemolytic streptococcus, while the remaining cases showed no bacterial growth. Among the 8 streptococcipositive cases, Streptococcus agalactiae, a group B streptococcus was detected in 5 cases, and Streptococcus dysgalactiae, a group $\mathrm{G}$ streptococcus was detected in 3 cases. All positive cultures were obtained from patients with lower-extremity lymphedema.

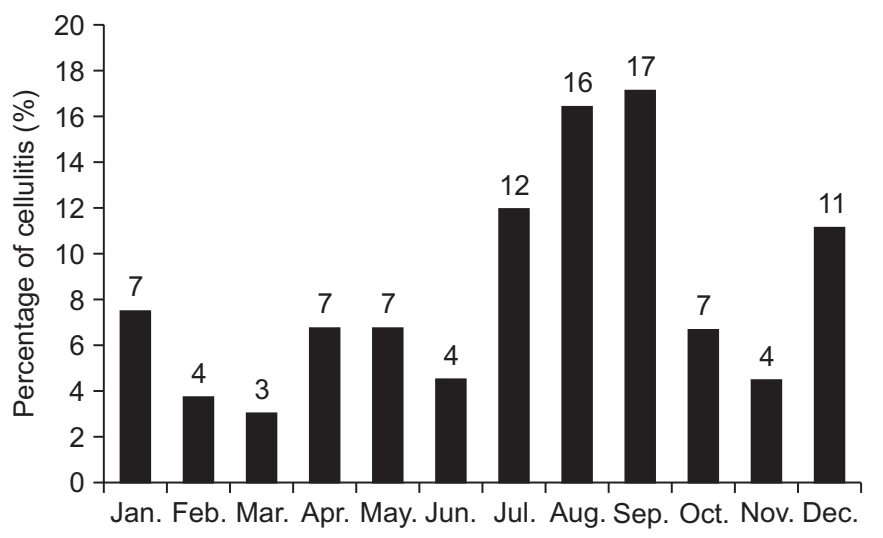

Fig. 2. Percentage of cellulitis ${ }^{\text {a) }}$ with lymphangitis ${ }^{\text {b) }}$ cases stratified by month. ${ }^{\text {a) }}$ Cellulitis (ICD-10 code: L039),

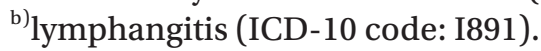

Average values for WBC count and ESR were not different between the patient groups when divided by the affected extremity. However, there was a significant difference in CRP level $(\mathrm{p}=0.006)$. On comparing cellulitis with lymphangitis episodes in patients who showed a value exceeding the normal range, the lower-extremity group (112 total episodes) had a significantly higher number of inflammatory responses in terms of WBC count values with 49 episodes compared to 19 episodes in the upperextremity group (76 total episodes) $(\mathrm{p}=0.030)$ (Table 2 ).

Most cellulitis with lymphangitis patients received intravenous third-generation cephalosporin, whose therapeutic effect was detected after an average of 9.5 days. Combination antibiotic therapy, such as cephalosporin with aminoglycoside was prescribed more frequently than monotherapy with vancomycin or cephalosporin. The attending physician changed the antibiotic course after the response to treatment was unsatisfactory in 10 cases; treatment-regimen changes occurred more frequently in lower-extremity lymphedema cases.

\section{DISCUSSION}

The prevalence rate of cellulitis including lymphangitis is variable across the globe. It is difficult to predict the occurrence risk because little epidemiological research has been performed, especially in Korea. Therefore, the objective of this study was to conduct an epidemiological investigation into the prevalence of cellulitis including lymphangitis among patients with lymphedema in Korea. We observed a regional cellulitis and lymphangitis preva- 
lence rate of $7.95 \%$ and a recurrence rate of $3.93 \%$. The prevalence and recurrence rates of cellulitis with lymphangitis were significantly higher in patients with lowerextremity lymphedema than in patients with upperextremity lymphedema. A previous retrospective study showed that the lower leg is more likely to be affected because of localization of lymphatic drainage [12].

General risk factors that are considered to have a close association with cellulitis risk include obesity, smoking, alcohol consumption, and diabetes mellitus [13]. In this study, the average BMI of patients with cellulitis including lymphangitis was $24.27 \mathrm{~kg} / \mathrm{m}^{2}$, which was categorized as overweight $\left(23-25 \mathrm{~kg} / \mathrm{m}^{2}\right)$. Although we did not conduct a statistical comparison between patients diagnosed with and without cellulitis in lymphedema groups, another study showed that obesity was linked to an increased risk [17].

Local factors causing defects of the skin barrier can increase the risk of developing cellulitis through invasion by microorganisms via an opportunistic pathway $[5,6,13]$. Skin trauma, lacerations, puncture wounds, leg ulcers, dermatitis, toe web maceration, and tinea pedis cause skin-barrier defects.

A history of cellulitis is known to increase the recurrence risk, possibly due to repeated damage to local soft and lymphatic tissue [11]. Risk from prior incidents is compounded by the presence of other risk factors [18].

Patients with lymphedema in their upper extremities were more likely to develop cellulitis with lymphangitis within 6 months of lymphedema onset, while patients with lower-extremity lymphedema were more likely to develop cellulitis with lymphangitis after 6 months of lymphedema onset. There was a significant association between increased time from onset and development of cellulitis with lymphangitis among the lower-extremity patient group. Several previous studies have shown that lymphedema itself is the strongest risk factor for cellulitis $[13,19]$, especially for recurrent cellulitis. On comparison with general edema secondary to venous insufficiency, it is known to have a particularly strong association with cellulitis in lower-extremity lymphedema. In an epidemiological study of a London-based population of 218 patients, $29 \%$ of lymphedema patients had experienced an episode of cellulitis within the previous 12 months [20].

Dupuy et al. [13] found that lymphedema was present in $18 \%$ of their patients affected with cellulitis involving
167 patients. A different study, in which patients who had more than two episodes of lower-extremity cellulitis were investigated with lymphoscintigraphy, found significant lymphatic abnormalities, thought to be related to infective episodes [21]. These findings are consistent with the conclusion that previously undetected lymphatic abnormalities may be the underlying factors in cellulitis cases. Early detection of lymphatic abnormalities through lymphoscintigraphy is regarded as the gold standard diagnostic approach, and early detection will enable more effective treatment to reduce and prevent future cellulitis episodes. However, there are also realistic limitations to effective early treatment, even when abnormalities have been clearly demonstrated.

It is widely known that the relationship between cellulitis and lymphedema is like a vicious cycle in which each episode of cellulitis causes further damage to the lymphatic system, causing some degree of secondary lymphedema, which in turn, increases the risk for cellulitis $[22,23]$. One study found that at least one episode of cellulitis or skin infection occurs in approximately $25 \%$ of lymphedema patients [24]. This cyclic relationship is independent of the primary etiology of lymphedema and is multifactorial. Protein-rich lymphatic fluid serves an excellent medium for the growth of many bacterial strains. Furthermore, stagnation of lymphatic fluid due to impaired lymph drainage accompanied by reduced lymphatic clearance can cause a decrease in local immunity, increasing the cellulitis risk $[25,26]$.

In patients without lymphedema, cellular material produced by bacteria is eliminated by phagocytosis and then it is effectively cleared through lymphatic drainage. However, in lymphedema patients, bacterial toxins in lymphatic tissue that cannot be drained sufficiently cause systemic symptoms due to cytokine release in response to the presence of excessive lymph fluid [25]. Once bacteria invade the edematous tissue, eradication can be difficult, and there is always a risk of cellulitis recurrence if the local immune system is impaired.

The eight blood cultures examined in this study that were positive for $\beta$-hemolytic streptococcus were obtained from patients with lower-extremity lymphedema. Similarly, a retrospective study found that only $2 \%$ of a 757-patient sample of community-acquired cellulitis cases was affected by a patient-specific microbial strain [27]. Another study reported that positive blood cultures 
were more common when cellulitis occurred after lowerextremity lymphedema [25]. Besides, in the laboratory findings to confirm differential inflammatory changes between the two extremity groups, there were no significant differences in most of the average values and cellulitis with lymphangitis episodes between the patients with upper and lower extremity lymphedema. Generally, it is difficult to identify inflammatory changes based on WBC count, ESR, or CRP in cases of cellulitis with lymphangitis, with the exception of severe cases that are accompanied by ulceration, blistering, or systemic symptoms, such as bacteremia or fever. Thus, most laboratory methods, including blood culture, have a relatively low diagnostic yield in cellulitis.

Once bacteria invade the edematous tissue, complete eradication is difficult, and there is always a risk of cellulitis reoccurrence if the local immune system is impaired. To inhibit these pathogenic processes, antibiotics with broad anti-streptococcal activity and immunomodulatory action are used. In our study, the evaluation of antibiotic treatments was limited to two hospital sites; hence, future studies should assess antibiotic treatment regimes in a larger, more diverse population at a broader scale because there is still no established treatment protocol, and the types of antibiotics used for treating cellulitis with lymphangitis are highly variable.

Some additional study limitations should be noted. First, we did not consider the underlying diseases that can interact with cellulitis, such as allergic dermatitis, diabetes mellitus, or tinea pedis. Second, our study did not include patients with severe symptoms, such as bacteremia with abnormality found in the laboratory findings. Third, we did not account for skin diseases, such as necrotizing fasciitis or erysipelas, other than lymphangitis. Fourth, we did not consider the relative risk for cellulitis according to the grade and stage of lymphedema, nor did we conduct an imaging analysis using methods like lymphoscintigraphy. Finally, this study was conducted retrospectively in only two regional hospitals and no comparison was made between patients diagnosed with and without cellulitis in lymphedema groups; thus, the results may not be generalizable to all rehabilitation centers.

In this study, there was high risk of cellulitis with lymphangitis in patients after occurrence of lower-extremity lymphedema. Cellulitis in lymphedema patients can worsen the ongoing edema despite continuous treatment; continued worsening increases the possibility of systemic infection, which further increases the need for immediately effective antibiotics to prevent exacerbation of the inflammatory response. An understanding of the risk for cellulitis will enable physicians to better counsel patients about the prognosis, and it will also facilitate further research on treatment.

\section{CONFLICT OF INTEREST}

No potential conflict of interest relevant to this article was reported.

\section{REFERENCES}

1. Smeltzer DM, Stickler GB, Schirger A. Primary lymphedema in children and adolescents: a follow-up study and review. Pediatrics 1985;76:206-18.

2. WHO Expert Committee on Filariasis. Lymphatic filariasis: fourth report of the WHO Expert Committee on Filariasis. World Health Organ Tech Rep Ser 1984;702:3-112.

3. Rockson SG, Rivera KK. Estimating the population burden of lymphedema. Ann N Y Acad Sci 2008;1131:147-54.

4. Godoy JM, Silva SH, Godoy MF. Sensitivity and specificity of combined perimetric and volumetric evaluations in the diagnosis of arm lymphedema. Prague Med Rep 2007;108:243-7.

5. Swartz MN. Clinical practice. Cellulitis. N Engl J Med 2004;350:904-12.

6. Cox NH. Management of lower leg cellulitis. Clin Med (Lond) 2002;2:23-7.

7. Damstra RJ, van Steensel MA, Boomsma JH, Nelemans P, Veraart JC. Erysipelas as a sign of subclinical primary lymphoedema: a prospective quantitative scintigraphic study of 40 patients with unilateral erysipelas of the leg. Br J Dermatol 2008;158:1210-5.

8. Masmoudi A, Maaloul I, Turki H, Elloumi Y, Marrekchi S, Bouassida S, et al. Erysipelas after breast cancer treatment (26 cases). Dermatol Online J 2005;11:12.

9. El Saghir NS, Otrock ZK, Bizri AR, Uwaydah MM, Oghlakian GO. Erysipelas of the upper extremity following locoregional therapy for breast cancer. Breast 2005;14:34751.

10. de Godoy JM, da Silva SH. Prevalence of cellulitis and erysipelas in post-mastectomy patients after breast can- 
cer. Arch Med Sci 2007;3:249-51.

11. de Godoy JM, de Godoy MF, Valente A, Camacho EL, Paiva EV. Lymphoscintigraphic evaluation in patients after erysipelas. Lymphology 2000;33:177-80.

12. Tsao H, Johnson RA. Bacterial cellulitis. Curr Opin Dermatol 1997;4:33-41.

13. Dupuy A, Benchikhi H, Roujeau JC, Bernard P, Vaillant L, Chosidow O, et al. Risk factors for erysipelas of the leg (cellulitis): case-control study. BMJ 1999;318:1591-4.

14. Shih YC, Xu Y, Cormier JN, Giordano S, Ridner SH, Buchholz TA, et al. Incidence, treatment costs, and complications of lymphedema after breast cancer among women of working age: a 2-year follow-up study. J Clin Oncol 2009;27:2007-14.

15. Teerachaisakul M, Ekataksin W, Durongwatana S, Taneepanichskul S. Diet, C-reactive protein levels and cellulitis in patients with lymphedema: a cross-sectional study. J Med Med Sci 2011;2:1297-1301.

16. Lewis SD, Peter GS, Gomez-Marin O, Bisno AL. Risk factors for recurrent lower extremity cellulitis in a U.S. Veterans Medical Center population. Am J Med Sci 2006;332:304-7.

17. Scheinfeld NS. Obesity and dermatology. Clin Dermatol 2004;22:303-9.

18. Bjornsdottir S, Gottfredsson M, Thorisdottir AS, Gunnarsson GB, Rikardsdottir H, Kristjansson M, et al. Risk factors for acute cellulitis of the lower limb: a prospective case-control study. Clin Infect Dis 2005;41:1416-22.

19. Duvanel T, Auckenthaler R, Rohner P, Harms M, Saurat
JH. Quantitative cultures of biopsy specimens from cutaneous cellulitis. Arch Intern Med 1989;149:293-6.

20. Moffatt CJ, Franks PJ, Doherty DC, Williams AF, Badger C, Jeffs E, et al. Lymphoedema: an underestimated health problem. QJM 2003;96:731-8.

21. Soo JK, Bicanic TA, Heenan S, Mortimer PS. Lymphatic abnormalities demonstrated by lymphoscintigraphy after lower limb cellulitis. Br J Dermatol 2008;158:1350-3.

22. Collins PS, Villavicencio JL, Abreu SH, Gomez ER, Coffey JA, Connaway C, et al. Abnormalities of lymphatic drainage in lower extremities: a lymphoscintigraphic study. J Vasc Surg 1989;9:145-52.

23. Woo PC, Lum PN, Wong SS, Cheng VC, Yuen KY. Cellulitis complicating lymphoedema. Eur J Clin Microbiol Infect Dis 2000;19:294-7.

24. Badger C, Seers K, Preston N, Mortimer P. Antibiotics/ anti-inflammatories for reducing acute inflammatory episodes in lymphoedema of the limbs. Cochrane Database Syst Rev 2004;(2):CD003143.

25. Baddour LM, Bisno AL. Non-group A beta-hemolytic streptococcal cellulitis: association with venous and lymphatic compromise. Am J Med 1985;79:155-9.

26. Mortimer PS, Levick JR. Chronic peripheral oedema: the critical role of the lymphatic system. Clin Med (Lond) 2004;4:448-53.

27. Perl B, Gottehrer NP, Raveh D, Schlesinger Y, Rudensky B, Yinnon AM. Cost-effectiveness of blood cultures for adult patients with cellulitis. Clin Infect Dis 1999;29:1483-8. 